\title{
DIGITALCOMMONS
}

—@WAYNESTATE-

Wayne State University

$1-1-2012$

\section{A Design-Based Research Case Study Documenting a Constructivist ID Process and Instructional Solution for a Cross-Cultural Workforce}

\author{
Monica W. Tracey \\ Wayne State University, Monicatracey@wayne.edu \\ Kelly L. Unger \\ Wayne State University, kellyunger@wayne.edu
}

\section{Recommended Citation}

Tracey, M. W., \& Unger, K. L. (2012). A design-based research case study documenting a constructivist ID process and instructional solution for a cross-cultural workforce. Instructional Science, 40(3), 461-476.

Available at: http://digitalcommons.wayne.edu/coe_aos/21 
A Design-Based Research Case Study Documenting a Constructivist ID Process and Instructional Solution for a Cross-Cultural Workforce

\author{
Monica W. Tracey \\ MonicaTracey@wayne.edu \\ Phone: 313-577-1700 \\ Wayne State University \\ 383 Education \\ Detroit, MI 48202 \\ Kelly L. Unger \\ KellyUnger@wayne.edu \\ Phone: 717-649-8545 \\ WSU College of Education IT Offices \\ 3 South Education \\ Detroit, MI 48202
}

\begin{abstract}
As the need for instructing a globalized workforce increases, instructional designers must embrace the constraints and the opportunities these projects provide in order to move the field of cross-cultural instructional design (ID) forward. Cross-cultural projects offer multiple avenues for growth in ID practice, overcoming cultural barriers, and a venue to apply and test contemporary models, methodologies, and theories in ID. This design-based research case study employed a rapid prototyping methodology and the constructivist ID model, Layers of Negotiation, to collect critical cultural information for the design of this cross-cultural instruction. Instructional strategies deemed effective for instructing an unskilled workforce in Dubai, United Arab Emirates, included job aids, situated learning and apprenticeship principles consisting of modeling, coaching and scaffolding. Use of the constructivist ID model and instructional solution for a cross-cultural workforce for The Dubai Mall are presented. Evaluation results indicate the success of the instructional strategies varied dependent upon worker culture, and the sequential nature of the instructional strategies.
\end{abstract}

Keywords: Cross-cultural instructional design; Design-based research; Job aids; Apprenticeship principles; Constructivist ID model; Layers of Negotiation Model 


\section{Background}

As the need for teaching members in global organizations increases, instructional designers are frequently tasked to design instruction for a cross-cultural workforce. Young (2008) states that, "the role of culture in Instructional Design (ID) has many meanings that inform learners and learning.... and these trends toward multiculturalism in ID should have designers rethinking what it means to integrate culture in design..." (p.7). Although the term culture has numerous meanings, in this case study culture is defined as language, religion, ethnicity, region of country, and gender of the worker. Designing instruction for a cross-cultural workforce often entails creating instruction for learners from cultures foreign to designers, requiring designers to quickly educate themselves on the cultural differences and the importance and impact these differences pose on the instructional design and delivery (Rogers, Graham, \& Mayes, 2007). Often designers lack the time and resources to educate themselves on the culture of the learners. In response to this constraint, designers ought to utilize contemporary models, methodologies and strategies to engage learners and other stakeholders during the design and delivery of instructional materials. Designers creating instruction for cross-cultural learners should embrace the contextual constraints and the opportunities these projects provide.

The purpose of this design-based research case study was to document the cross-cultural iterative ID process and ID solution ${ }^{1}$, and to evaluate the results of this solution in training a cross-cultural workforce. The results of this case assist in moving the practice of using constructivist ID principles for designing cross-cultural instruction forward.

\section{Case Background}

\footnotetext{
${ }^{1}$ The ID processes and issues regarding this project have already been published and can be found at: http://scholarworks.iu.edu/journals/index.php/ijdl/article/view/845/909
} 
A large international real estate development firm determined a need to ensure that one of their client's properties, The Dubai Mall (TDM) would be the cleanest mall in the world. This firm sought out a university-based instructional design (ID) team to design instruction for a cross-cultural workforce that would be tasked with cleaning this mall. The US based ID team, a US based cleaning company, and real estate representatives traveled to Dubai, United Arab Emirates (UAE) to meet with the client and conduct an initial needs assessment.

Initial assessment data indicated that TDM needed employee and supervisory staffing and training of just over 400 workers to effectively achieve the goal of being the cleanest mall in the world. This staff was recruited from countries including Bangladesh, India, Nepal, and the Philippines. The workers were considered unskilled workers. The mall environment lacked existing instructional materials, technology, and a performance review process. Furthermore, workers lacked knowledge of use of job materials, skills in performing the job procedures, and had an inability to read and speak a common language. In addition to the worker and environmental constraints, the instructional design team faced a severe seven week design and implementation time constraint due to the client's requisite of effective and efficient cleaning procedures and effective worker performance the day the mall opened. In order to increase the probability of knowledge to performance transfer, the ID team determined the training of the entire workforce must occur in the TDM work environment.

\section{Methodology}

Results from the initial needs assessment, the naturalistic nature of the project, the evergrowing importance of cross-cultural instruction in a constantly shifting globalized world, and the ID team's lead project manager/researcher role indicated this case was conducive for employing a design-based research case study. 


\section{Design Process}

Due to the severe ID time constraints and complex nature of the project, the ID team needed constant access to all of the stakeholders in the project in an effort to produce instructionally accurate materials. This project, while critical in a cross cultural training environment, but common of ID projects with little budget and time, did not allow the necessary time to fully understand the learner or work environment, because expatriate workers had not yet arrived in Dubai, and the Mall construction was incomplete. In order to achieve access to stakeholders, the team submerged themselves in the contextual environment of the instructional location, The Dubai Mall (TDM) throughout the design, development and implementation process. The ID team arrived in Dubai and immediately connected with client stakeholders, the cleaning chemical and equipment companies, and TDM management staff to develop task lists for all of the equipment and cleaning procedures. The next step was to learn about the workers.

\section{Participants.}

Residents of the UAE make it one of the most diverse populations in the Middle East (Kaleel \& Cattarossi, 2008), requiring training programs "to be tailored to the diverse makeup of the team, with several considerations for those of Muslim faith, since 96 percent of the population practices Islam" (p.20). Dubai has always been considered a merchant city, and for decades a major hub for boats heading to and from Persia, India, China, and Africa (Al-Sayegh, 1998). In the 1970's oil had been discovered and provided extreme wealth to Dubai's citizens, who began wanting more elaborate buildings and lifestyles, but did not want to do the manual labor. Today the workforce in the UAE is comprised of approximately 3.2 million foreign workers (AMEinfo, 2009). Many of these workers are unskilled and have had very little if any education. The workers for TDM came from small villages and had limited to no prerequisite 
knowledge of the materials and environment they were to clean. Analysis and observation of worker characteristics, previous experience, and identified job procedures, aided in determining what instructional strategies were needed in the design.

In order to implement a culturally sensitive customized instruction plan to increase worker skills, and reduce the language and cultural barriers, it was necessary to research the demographics of each workgroup. The workforce consisted of four cultures discussed in the following section: Bangladesh, India, Nepal, and Philippines.

The majority of the Bangladesh labor force work in agriculture (63\%) and the service industry (26\%) (US State Department, 2010). Although the major languages include Bangla and English the total adult literacy rate is $47.5 \%$. The major religions of Bangladesh workers are Muslim (83\%) and Hindu (16\%).

India's labor force is quite similar to that of Bangladesh, with most occupying employment in agriculture (60\%) and service jobs (28\%) (US State Department, 2010). India's major languages include Hindi, English, and sixteen other official languages with a total literacy rate of $61 \%$. Major religions are Hindu (81.4\%) and Muslim (12.4\%).

The labor force in Nepal employs workers mostly in agriculture (71\%) and the service industry (11\%) (US State Department, 2010). Languages spoken in Nepal include, Nepali and over 100 other regional and indigenous languages, and have a total literacy rate of $49 \%$. The major religions in this country are Hindu (81\%) and Buddhist (11\%).

Of the four cultures discussed, the Filipino labor force employs the highest percentage of its people in the service industry (51\%) followed by agriculture (34\%) (US State Department, 2010). The country's major languages are Filipino and English, with a 93.4\% literacy rate. Roman Catholicism is the major religion practiced (80.9\%). These cultures encompassed the 
unskilled labor force tasked with cleaning The Dubai Mall. In addition, supervisors of these workers were also predominantly from these four cultures.

\section{Methodological and theoretical foundations.}

The ID team researched ID and cultural competence applying guiding principles applicable to this design when possible. However, the premise of this study was to examine constructivist ID principles for designing cross-cultural instruction. Therefore, a rapid prototyping methodology and the constructivist ID principles of the Layers of Negotiation Model were the foundations framing this study.

Rapid prototyping and Layers of Negotiation Model. The ID team determined that in an effort to ensure success in this project while meeting client expectations, under the environmental, worker, and time constraints, they needed to employ the rapid prototyping methodology and the constructivist ID Layers of Negotiation Model (Cennamo, 2003). The Layers of Negotiation Model used for the design process, involves reflecting and examining information from multiple perspectives by all those involved in the design at multiple times during the design process (Richey, Klein \& Tracey, 2011). Rapid prototyping is an ID methodology in which designers work with clients to quickly build a series of prototypes for instruction before committing to a single instructional approach for further development (Bichelmeyer, 2004). This methodology allowed for reduced development costs and time, decreased communication problems and produced the right system for the tasks (Tripp \& Bichelmeyer, 1990).

Rogers et al. (2007) found three barriers designers have in their effort to design more culturally responsive instruction: 
(a) an over emphasis on content development as the center of practice and under emphasis on context and learner experience, (b) a relative lack of evaluation in real-world practice, and (c) the creation of less than ideal roles that instructional designers assume in the larger organizational structures involved (Rogers et al., pp.206-207).

To overcome these potential barriers the ID team worked in TDM offices during the design, development, pilot, and evaluation of the instructional materials. Immersing the ID team directly in the facility assisted the team in gaining a better understanding of the learner and contextual environment through daily access to all stakeholders including Mall management, supervisors, and workers. The ultimate goal was a successful mall opening and an ongoing clean mall, and due to the ongoing participation of all stakeholders throughout the design process, the ID team's role was understood and valued as an integral part of achieving that goal.

Terry (2007) when documenting a case study of motivating a multicultural workforce identified certain ways to approach workers who do not have English as their primary language, as was the case with this project. These include awareness of communication barriers, building trust with the workers, and an understanding of how different cultures view leadership and decision making. The immersion and collaboration with the design team and the executive stakeholders created trust with the stakeholders and the ID team. These stakeholders provided iterative feedback during the design of the instructional materials and instructional strategies which attempted to address worker communication barriers while building worker trust and respecting the worker's view of leadership.

The significant cultural differences between the workers and what they brought to the learning experience, along with environmental and time constraints impacted the approach of the design. It required ongoing input from content and cultural subject matter experts, workers, 
supervisors, and stakeholders throughout the process. This input assisted in an efficient and thorough process of collecting necessary worker cultural data that was not possible during a traditional needs assessment. Upon arrival in Dubai, The ID team implemented the Layers of Negotiation Model, a constructivist ID model that employs all stakeholders in a project and focuses on the process of knowledge construction using reflection, the examination of information multiple times for multiple purposes, and the social negotiation of shared meanings (Cenamo, 2003). One output of implementing this model was daily update stakeholder gatherings. These meetings provided a venue for all stakeholders to make instant decisions and revisions of process, product and instructional materials. During one gathering for example, the cleaning subject matter expert chose a mop to clean common surface areas. The ID team initiated initial design procedures to train workers to use this mop. During the following day's gathering, the cleaning material supplier informed the stakeholders the mop could not be shipped to the UAE, thus altering the materials and the instruction. As the project unfolded, the design team and researcher were in a unique situation to participate in and document the complex issue of designing this cross-cultural project. Due to the numerous project constraints, the Layers of Negotiation Model provided the framework necessary to require participatory design by all involved in the project (Cennamo, 2003).

Design-based research. Incorporating the rapid prototyping methodology and the Layers of Negotiation Model permitted iterative cycles of design and observation while creating instructional materials (Statwicz \& Stevens, 2008), that would impact both the local needs of TDM, as well as test the application of applying constructivist ID principles. The focus of design-based research is on studying design in practice (Barab \& Squire, 2004). This case tested the application of theoretical constructivist ID principles placing the researcher, also the lead 
project manager, in the role of an applied researcher whose goal was "to directly impact practice while advancing theory that will be of use to others" (Barab \& Squire, 2004, p.8). Wang and Hannafin (2005) describe the researcher's role in design-based research as one where "researchers manage research processes in collaboration with participants, design and implement interventions systematically to refine and improve initial designs, and ultimately seek to advance both pragmatic and theoretical aims affecting practice" (p.6). Immersing the ID team in the environment and working collaboratively with all stakeholders provided the researcher/lead project manager access to improve the design of instruction, and apply constructivist ID principles that affected cross-cultural ID practice.

\section{Culture and a cultural subject matter expert.}

Webster (1998) defines culture as "the customary beliefs, social forms, and material traits of a racial, religious, or social group" (p.282). For this case, culture is defined as language, religion, ethnicity, region of country, and gender of TDM workers. Understanding the environmental culture as well as the cultures of the workers was critical to the successful design of the instruction. Designers bring preconceived design notions to each project, so the initial analysis was approached with an instructional designer who was also a cultural expert from the Middle East. His presence and expertise in the initial phase of this design-based research project was critical as it shortened the design team's learning curve and assisted in making informed design decisions for this diverse population, under the project's time constraints. The cultural expert assisted in explaining the assumptions the design team could and could not make regarding the learners and the instructional materials. One example was in the use of symbols in the instructional materials. Symbols are used and interpreted differently in different cultures. The cultural expert informed the team of what symbols could be used and how each would be 
interpreted. Due to Islamic beliefs for example, it is inappropriate to use a graphic of the full body, therefore only the hands were used to illustrate cleaning tasks.

\section{The Design Solution}

Results of the initial needs assessment, immersion by the ID team in the mall environment, implementation of rapid prototyping and the Layers of Negotiation Model, provided the team direction in the initial design solution of a standardized cleaning system and a cross-cultural customized instructional program. The ID team needed to provide cross-cultural customized instruction that utilized concrete terms, simple language, familiar shapes, and colors. The ID solution included instructional materials that employed a combination of job aids and apprenticeship principles.

\section{The design and implementation of instructional strategies.}

The job of the ID team was to design training to teach a cross-cultural and unskilled workforce a systematic process to clean the mall using supplies many of the workers had never seen or used. Instructional materials needed to be developed in order for workers to learn and correctly perform all job functions. It was also necessary that the materials were designed for supervisors to fully understand every process in order to replicate and reinforce instruction after the ID team completed the project and left Dubai.

Use of job aids. DeBry (2002), when studying seven different global companies, found job aids to be one form global companies were using in designing instructional materials and distributing them to their employees. Job aids were selected as an instructional strategy in order to overcome the language and cultural barriers, and to assist the worker in completing the procedures while on the job. 
Using ID principles, the instructional solution consisted of detailed customized instruction that eliminated the language barrier. The first step in designing the job aids was to work with the cleaning subject matter experts to document the thirteen job procedures. Once the procedures were documented, the ID team created a number of job aid prototypes utilizing a combination of color, symbol and graphic representation of each procedure as it needed to be performed. At this stage of the design process, through incorporating feedback from all stakeholders, the ID team was able to quickly modify the job aids. For example, as the engineers from the cleaning company made path changes in the cleaning process, these path changes affected the steps in the instruction, which ultimately altered the graphics in the job aids. The job aids were also used to assist in achieving an additional organizational goal; that all workers eventually learn the English language. When incorporating written instruction in the job aids, the words were depicted in English and then translated into Hindi, pulling the workers' previous knowledge of Hindi words, using pictures, colors, and symbols to describe the job procedures, and then supplied them with the English words (See Figure 1).

Insert Figure1 about here

This form of instruction served a multiple purpose: it instructed how to perform cleaning tasks, while introducing the worker to the English language. The goal was to use this approach to build worker confidence to interact with supervisors and over time help reduce the language barriers. The instructional process was designed in that after the initial implementation of the instruction to the workers by the ID team, the supervisors would use the detailed job aids to continuously train the workers. 
Each job procedure was comprised of sub-tasks. The designers created the job aids to assist in visually illustrating the step-by-step sub-tasks workers needed to perform in order to complete the entire job procedure. The workers needed instructor modeling of each sub-task and direction on how to use the job aids. The majority of the workers were unfamiliar with general cleaning and the mall environment was alien. Due to the lack of knowledge, use of the English language, and lack of skill, the ID team determined the best approach to training was one that demonstrated desired behavioral performance of the job procedures and utilized the job aid.

Situated learning and apprenticeship principles. The instructional materials were designed to be delivered in three cycles to the workers in the mall environment where workers could actively learn and perform the procedures they would be required to employ. Workers learning by doing and interacting with the job procedures in the mall environment is an example of situated learning (Brown, Collins, \& Duguid, 1989), founded on the belief that this approach promotes higher transfer of skills than traditional information-dissemination training (Dennen, 2003). The instructional designers also used apprenticeship principles, often found in cognitive apprenticeships including, modeling, explanation, coaching, and scaffolding (Jonassen, 1999; Enkenberg, 2001; \& Dennen, 2003). A typical apprenticeship approach includes an expert working with a novice, and as the novice gains more experience of the specific task or process the expert fades, scaffolds, or slowly withdraws from the process so the novice can complete the task or process on their own (Brown et al., 1989; Dennen, 2003). This training was designed for the first instructional cycle to include one facilitator demonstrating (model) each sub-task for each job procedure while another simultaneously stated (explained) the sub-task while showing the worker the job aid. The worker actively followed the two facilitators by completing the subtasks as they were modeled. Modeling, in this case, is a form of behavioral modeling, and is one 
of the most effective ways to train psychomotor skills because of the workers active imitation of the performed task (Dennen, 2003).

The second cycle of instruction which occurred immediately after the first, included workers performing the job procedures on their own without the use of the facilitator modeling; the facilitators' transitioned to the role of observer and the worker relied on the use of the job aid alone for completing each task. The traditional apprenticeship model, which typically has the expert slowly withdrawing from the instructional process, was modified in this case due to time constraints.

By combining these two instructional strategies, the use of job aids and basic principles of apprenticeship, the intent was to have workers completing their job procedures completely from memory during the third cycle of instruction. Williamson states "Using an apprenticeship system, which involves learning-and teaching-by doing, are often the most efficient means of communication" (as cited in Carayannis \& Jorge, 1998, p.385). The apprenticeship principles provided a strategy for designing the initial instruction, and created an environment to easily incorporate another principle, coaching. Coaching involves the long-term monitoring of a novice performance by an expert who can provide motivational prompts (Jonassen, 1999) and guidance for assisting the novice when necessary (Enkenberg, 2001). The ID team incorporated the coaching principle for the supervisors to use with the workers after the initial instruction.

\section{Data Collection and Analysis}

Six instructional facilitators ( 2 men and 4 women) were recruited from the United States to implement the apprenticeship approach. They were selected based on their expertise in facilitation of instruction including demonstrating tasks and modeling correct performance of on the job skills. Prior to traveling to the UAE, the facilitators successfully completed a one week 
concentrated instructional course on systems cleaning, and very quickly became knowledgeable of cleaning tasks. Another component to assist the facilitators was the presence of two cleaning SMEs in the instructional environment who observed and provided continuous feedback to the facilitators after the delivery of each instructional event. Although time constraints hindered the possibility of the facilitators becoming cultural experts, each were required to study cultural information packets provided prior to their arrival in Dubai.

The facilitators delivered the instruction to 40 supervisors and 375 cleaning workers during a three week timeframe. Thirteen total teams were staffed to populate the Mall cleaning system. This posed an additional constraint as the teams were not formed based on cultural similarities rather based on the needs to operate the cleaning system. Therefore the direct supervisors, who simultaneously cleaned and directed the unskilled workers, may or may not have come from the same four countries as the workers. The supervisors had varying degrees of literacy and command of the language providing additional rationale to remove verbal language as much as possible in the instruction. Direct supervisors and unskilled workers were aligned by gender although most workers were men, with the exception of women teams created to clean the Mall's women's washrooms and abolition rooms.

The team of facilitators ultimately trained and evaluated employees on thirteen procedures. Evaluation sheets were developed for each of the thirteen procedures, and were color-coded to coincide with the job aids for each procedure (See Figure 2).

Insert Figure2 about here 
The evaluation sheets were designed to allow facilitators the opportunity to evaluate the workers performing the tasks during the three different cycles while: (1) facilitators modeled and explained each sub-task with the job procedures as the worker performed the task with the use of job aids, (2) worker performed tasks with job aids only, and (3) worker performed the sub-tasks from memory without any assistance. The facilitator marked Yes or No to indicate whether or not the worker was able to complete the task during each of the three attempts. Facilitators could also mark language, equipment, supplies, learning path, and other, as issues for hindering successful completion of each sub-task.

The evaluation sheets were completed on the job site by hand using pen and pencil while the workers performed the sub-tasks. Evaluations were collected and grouped together by the thirteen job procedures, and then compiled into a digital spreadsheet. Since each procedure had a different number of sub-tasks, each sub-task had to be entered for each of the three cycles in order to determine which instructional strategy proved to be most successful. Due to the varying number of sub-tasks (ranging from 24-166, with an average of 64) within each job procedure, the first step was to calculate the total number of tasks completed by each participant and then calculate the relative achievement score ( $\%$ passed dependant on the number of tasks performed). This data was then placed into SPSS to analyze which instructional strategy; modeling with job aid, job aid only, or from memory demonstrated the most significance.

Rater agreement coefficient was not computed because there was no variation, or underlying continuum, between the instructors evaluation of each sub-task. The response to the workers performance could only be Yes or No identifying if the worker completed the sub-task or not. Both responses in this case were dichotomous and running these tests is typically only necessary when one variable is dichotomous, not both (Fields, 2005). 


\section{Results}

This section describes data results of the three instructional strategies, modeling with job aid, job aid only, and memory, compared to country, and any effects that each instructional strategy had on the next strategy.

\section{Measuring Instructional Strategy by Country}

A MANOVA test was employed because it is more robust when measuring several dependent variables rather than treating each one separately (Field, 2005). In this test the dependent variables were the instructional strategies and the fixed factor was the country. Running a MANOVA yielded all possible or customized contrasts between the instructional strategies. The Pillai trace test result revealed significant difference $(\mathrm{F}=4.144, \mathrm{df}=9, \mathrm{p}<.01$, $\eta 2=.087)$. The result of these tests demonstrates country differs significantly with respect to instructional strategies modeling with job aids, and job aid only (See Table 1). However, this does not hold true for the instructional strategy, performance from memory.

Insert Table 1 about here

\section{Comparing Worker Performance}

Three different univariate ANOVAs were performed for each instructional strategy for follow-up to MANOVA. The main purpose of these tests was to show the comparison of the countries' performance. To test for assumptions of equality between groups, a Box M's test was computed $(\mathrm{F}=27.727, \mathrm{df}=18, \mathrm{p}<.01)$; it compares the variance-covariance matrices between the groups (Field, 2005). Box M's test in this case showed significance, meaning the matrices are not the same. The significant Box M's test of equality, showing the group sizes are different, led to the need to look at post hoc tests. Follow-up post hoc tests were conducted, and the Dunnett's 
$\mathrm{C}$ procedure was chosen because it assumes unequal variances across groups to determine statistically significant differences between the pairs of the countries.

The modeling with job aid strategy results revealed significant difference between the countries $\left(\mathrm{F}=8.44, \mathrm{df}=3, \mathrm{p}<.001, \eta^{2}=.162\right)$. The effect size is at a large level (Cohen, 1988), and it explains $16.2 \%$ of the variance. The job aid only strategy results revealed significant difference between the countries $\left(\mathrm{F}=7.86, \mathrm{df}=3, \mathrm{p}<.001, \eta^{2}=.152\right)$ showing a large level effect size, explaining $15.2 \%$ of the variance. The results from the memory only strategy revealed significant difference between the countries $\left(\mathrm{F}=4.01, \mathrm{df}=3, \mathrm{p}<.01, \eta^{2}=.084\right)$. The effect size for these results is at a medium level, and explains $8.4 \%$ of the variance. Table 2, Table 3 , and Table 4 illustrates each post-hoc test results.

\section{Insert Table 2 about here}

\section{Modeling with job aid.}

The Philippine workers, when the instructional strategy modeling with job aid was used, performed significantly better than India. When compared with the other countries they performed better, but results are not significant. Bangladeshi's performed better than the Indian and Nepal workers, but results are only significant over Indian workers. Nepalese workers performed better than Indian workers, but results are not significant.

Insert Table 3 about here

\section{Job aid only.}


When comparing the countries performance using job aid only as the instructional strategy, the Filipino workers performed better than the other three countries, but results are only significant over Indian and Bangladeshi workers. Results show Bangladeshi workers performing better than Indian and Nepalese workers, but results are not significant. Nepalese workers performed better than Indian workers, but results are not significant.

Insert Table 4 about here

\section{Memory only.}

Even though this instructional strategy did not show significant results in the MANOVA test, the result of the ANOVA for this strategy is presented here. When comparing the countries using the memory only instructional strategy, the Philippine workers performed better than all three countries, but results are only significant over Indian workers. Nepalese workers performed better than the Indian and Bangladeshi workers, but results are only significant over Indian workers.

In the first analysis we assumed that the sequential nature of how the instructional strategies were implemented had no influence on the worker's performance by country. Therefore, an initial ANCOVA analysis was conducted to examine the sequential nature of the strategies impact on worker's performance. Since the modeling with job aid was the first instructional strategy applied before the other two, an ANCOVA was used for analyzing the job aid only strategy and country while controlling the modeling with job aid strategy's impact. The result of this analysis revealed that there is a significant impact of the modeling with job aid strategy on the job aid only strategy $\left(\mathrm{F}=20.17, \mathrm{df}=1, \mathrm{p}<.001, \eta^{2}=.134\right)$. The country also had a significant impact on the job aid only strategy $\left(\mathrm{F}=4.03, \mathrm{df}=3, \mathrm{p}<.01, \eta^{2}=.085\right)$. 
A final ANCOVA was conducted for analyzing the impact of country on the memory only strategy by controlling the outcomes of the two previously implemented strategies, modeling with job aids and the job aid only strategy. The result of the analysis revealed that both modeling with job aids $\left(\mathrm{F}=12.63, \mathrm{df}=1, \mathrm{p}<.01, \eta^{2}=.089\right)$ and job aids only $(\mathrm{F}=104.55, \mathrm{df}=1$, $\mathrm{p}<.001, \eta^{2}=.448$ ) had a significant impact on the third instructional strategy, memory. Nevertheless, the country did not yield a significant difference on the memory only strategy $\left(\mathrm{F}=.901, \mathrm{df}=3, \mathrm{p}>.05, \eta^{2}=.021\right)$, proving the instructional strategy sequence surpasses the country's impact on workers' performance.

\section{Discussion and Conclusion}

When motivating a multicultural workforce, Terry (2007) identified certain ways to approach workers who do not have English as their primary language. He indicated the importance of awareness of communication barriers, building trust with the workers and understanding how different cultures view leadership and decision making. One of the goals of job aids and apprenticeship principles in this project was to address some of these issues. The first two instructional strategies, modeling with job aid and job aid only, had a significant impact on the worker's performance, but this was not the case when workers performed the cleaning tasks from memory alone. This could be explained through trust issues which stemmed from the workers own fears; fears of authority, of being reprimanded or deported for doing something wrong, or of not having the modeling with job aid available for support and having to complete the task on their own. Time constraints, coupled with their own difficulty developing trust among the team, because they themselves were not savvy in building trust due to their cultural background and experiences, delayed the development of trust and communication between the supervisors and workers. Incorporating the multiple cycles of three instructional attempts for 
each procedure may have increased worker behavior and ultimate performance from memory, if instructional activities that specifically focused on building trust between supervisor and workers were implemented prior to implementing the cleaning instruction. Another option, if time permitted, may have included training the supervisors on the cleaning procedures first so they could co-facilitate the instruction. These options may have built leadership in the supervisors, in turn increasing their ability to build trust among their workers, but were not feasible in this context due to the time and environmental constraints.

The number of workers for each of the four countries in this project varied, so comparing the instructional strategies between the countries was imperative. When looking at the effect size, which shows the variance within the comparison groups, the results show a change in a certain amount of the workers ability to perform the tasks by using each of the three strategies. The results of each country's performance using each instructional strategy, shows that the Filipino workers performed better than the others, but not significantly in each comparison. A reason for the higher levels of success among the Filipino workers could be contributed to their higher literacy rate, which is approximately $40 \%$ higher than the other countries (US State Department, 2010). They also lead the other countries in percentage of workers that employ the service industry (51\%), while the other countries mainly work in agriculture. This may have provided these workers with additional perquisite knowledge of cleaning procedures and equipment. Another reason could be attributed to the Filipino workforce sharing the English language and many of the same cultural beliefs and values of that with the facilitators. Because these cultures are so varied even within their country, this study cannot infer generalizations about the cultural variations. However, one common value that all the workers shared was to earn an income to send back to their families in their home countries and to stay gainfully 
employed in the UAE. Every worker wanted to work hard and overcome any barrier that would prevent their success.

In this project, two factors, the instructional tasks and sequential order of the instructional strategies impacted worker performance. Regardless of the worker's country of origin, the simplicity of the tasks in this instruction, as compared to high-order complex cognitive tasks, may have caused the small performance differences with workers between each of the countries. When comparing the sequence of the instructional strategies with the worker country of origin, the sequential order of the strategies had a greater impact on worker performance than did the country. The sequence of the instructional strategies may have had a greater impact than the worker country of origin because of the overall lack of knowledge and skill all of the workers possessed independent of where they came from. The authors recognize these two factors as possible limitations of the study, and suggest that further research be conducted in different contextual settings, with high-order complex tasks, as well as replicating this study.

The work environment also posed limitations on the instructional performance of the workers. The first limitation was the temperature which was extremely hot, approximately 120 degrees Fahrenheit due to lack of air conditioning at the instructional site. This affected the workers who were required to wear heavy overalls. The second limitation was the construction of the Mall which was not complete. As a result, the training was interrupted by construction alarms causing facilitators and workers to wait until the alarms were shut off in order to resume instruction. The decision to conduct on-the-job instruction in this case was necessary in overcoming the identified lack of pre-requisite knowledge and experience however a performance environment that is physically conducive to on-the-job instruction is critical. 
When designing cross-cultural instruction, some researchers recommend incorporating culture based models and methods (Young, 2008a; Moalosi, Popovic, \& Hickling-Hudson, 2010; Henderson \& Lee, 1997). The purpose of these models is to assist instructional designers in creating and/or adapting instructional materials incorporating cultural information in the design. These cultural models and methods however were not employed in this context for two reasons. First, there was no need to include cultural information directly in the design of the instruction due to the content matter; cleaning is universal. Although cultural sensitivity was important (as described above in the cultural SME section), incorporating specific cultural information was not a necessary component of the job aid. Second, due to the severe time constraints resulting in limited data from the needs assessment the design team needed to employ other models and methods to collect information to design this cross-cultural project. The team therefore employed and documented the results of the Constructivist ID Layers of Negotiation Model and rapid prototyping methodology.

These two frameworks promoted the inclusion of all stakeholders throughout the entire project. For example, the participatory process resulting from the use of the Layers of Negotiation Model provided an opportunity for continuous cultural education throughout the design and development process. One instance of this was the initial use of the word "Bathroom" in our instruction. During one of the morning stakeholder gatherings it was discovered that the culturally preferred word was "Washroom." The significant factors in the process of this project included the ongoing participation of the ID cultural expert and the entire team of stakeholders which occurred as a result of employing the Layers of Negotiation Model. One goal of designbased research is to systematically understand and predict how learning occurs within the context in which it occurs. This goal was achieved during this project with the ID team. Identifying and 
documenting the process and results of the use of the Layers of Negotiation Model and rapid prototyping in this context contributes to the knowledge base of Constructivist ID.

Although this project presented numerous constraints including time and cultural barriers, documenting the design process and instruction for a cross-cultural unskilled workforce is significant for ID. The results from this study provide suggestions and considerations for other instructional designers who may apply constructivist ID principles in their cross-cultural instructional projects. As Terry (2007) describes, "The differences between the cultures that make up these workforces present new issues but, more importantly, new opportunities" (Terry, p. 59). This case illustratres one example of an ID team's process and product for training a cross-cultural unskilled workforce. As globalization continues to increase, so will these opportunities.

\section{References}

Al-Sayegh, F. (1998). Merchants' role in a changing society: The case of Dubai, 1900-90. Middle Eastern Studies, 34(1), 87-102.

AMEinfo (Wednesday, January 07 - 2009). UAE multinational workforce reaches 3.2 million in 206,000 firms. Retrieved from http://www.ameinfo.com/180506.html

Barter, J., Jette, C., \& Wiseman, D. (2003). Dancing numbers: Cultural, cognitive, and technical instructional perspectives on the development of Native American mathematical and scientific pedagogy. Educational Technology Research and Development, 51(2), 87 - 97.

Barab, S., \& Squire, K. (2004). Design-based research: Putting a stake in the ground. The Journal of the Learning Sciences, 13(1), 1-14. 
Bichelmeyer, B. (2004). Rapid prototyping. In A. Kovalchick \& K. Dawson (Eds.), Education and technology: An encyclopedia, Volume 2: J-Z (pp. 483 -489). Santa Barbara, CA: ABC CLIO.

Brown, J. S., Collins, A., \& Duguid, P. (1989). Situated cognition and the culture of learning. Educational Researcher, 18(1), 32-42.

Carayannis, EG (1998). Bridging government-university-industry technological learning disconnects: a comparative study of training and development policies and practices in the US, Japan, Germany, and France. Technovation, 18(6-7), 383.

Cennamo, K. (2003). Design as knowledge construction: Constructing knowledge of design. Computers in the Schools, 20(4), 13-35.

Cohen, J. (1988). Statistical power analysis for the behavioral sciences (2nd ed.). New York: Academic Press.

DeBry, D.P. (2002). Analysis of emerging practices in globalizing instruction materials. Educational Technology Research and Design, 50(4), 73-82.

Dennen, V. P. (2003). Cognitive apprenticeship in educational practice: research on scaffolding, modeling, mentoring, and coaching as instructional strategies. In D. H. Jonassen (Ed.), Handbook of Research on Educational Communications and Technology, (2nd ed.) (pp. 813-828). Mahwah, NJ: Lawrence Erlbaum, Inc.

Enkenberg, J. (2001). Instructional design and emerging models in higher education. Computers in Human Behavior, 17, 495-506.

Field, A.P. (2005). Discovering statistics using SPSS : And sex, drugs and rock ' $n$ ' roll (2nd ed.). Thousand Oaks, CA: Sage Publications. 
Henderson, L. (2007). Theorizing a multiple cultures instructional design model for e-learning and e-teaching. In A. Edmundson (Ed.), Globalized e-learning cultural challenges (pp. 130-153). Hershey, PA: Idea Group, Inc.

Jonassen, D. H. (1999). Designing constructivist learning environments. In C. M. Reigeluth (Ed.), Instructional-design theories and models: A new paradigm of instructional theory (pp. 215-239). Mahwah, NJ: Lawrence Erlbaum.

Kaleel, S. \& Blanchard-Cattarossi, K. (2008). Focus on UAE. Training, 45(7), 20.

Lee, C.D. (1997). Bridging home and school literacies: A model of culturally responsive teaching. In J. Flood, S. B. Heath \& D. Lapp (Eds.), A handbook for literacy educators: Research on teaching the communicative and visual arts (pp. 330-341). New York, NY: Macmillan.

Melkman \& Trotman (2005). Training International Managers: Designing, Deploying and Delivering Effective Training for Multi-Cultural Groups. Aldershot, United Kingdom: Gower Publishing.

Merriam-Webster (1998). Collegiate Dictionary (10 ${ }^{\text {th }}$ Ed) (p. 282). Springfield, MA: MerriamWebster, Inc.

Moalosi, R., Popovic, V., \& Hickling-Hudson, A. (2010). Culture-oriented product design. International Journal of Technology Design Education, 20 (2), 175-190.

Richey, R., Klein, J., \& Tracey, M. (2011). The instructional design knowledge base: Theory, research, and practice. New York: Routledge.

Rogers, P.C., Graham, C.R. \& Mayes, C.T. (2007). Cultural competence and instructional design: Exploration research into the delivery of online instruction cross-culturally. Educational Technology Research and Development, 55(2), 197-217. 
Statwicz, T. \& Stevens, R. (2008). A distributed perspective on collaborative activity. In M. Spector, M.D. Merrill, J. van Merrienboer, and M. Driscoll (Eds.), Handbook of Research on Educational Communications and Technology, (3 ${ }^{\text {rd }}$ ed.) (pp. 163-171). Mahwah, NJ: Lawrence Erlbaum, Inc.

Terry, J. (2007). Motivating a multicultural workforce. Instructional and Commercial Training, $39(1), 59-64$.

Tracey, M.W., \& Unger, K.L. (2010). Cross cultural instruction: An instructional design case. International Journal of Designs for Learning, (1)1.

Tripp, S. \& Bichelmeyer, B. (1990). Rapid Prototyping: An Alternative Instructional Design Strategy. Educational Technology Research and Design. 38(1), 31-44.

The U.S. State Department. U.S. Department of State: Diplomacy in action: Background Notes Retrieved from http://www.state.gov/r/pa/ei/bgn/index.htm

Young, P. (2009). Instructional design frameworks and intercultural models. Hershey, PA: Information Science

Young, P. (2008). Integrating culture in the design of ICTs. British Journal of Educational Technology, 39(1), 6-17.

Young, P. (2008a). The culture based model: Constructing a model of culture. Educational Technology \& Society, 11(2), 107-118. 